\title{
Conceptual design and modeling of particle-matter interaction cooling systems for muon based applications
}

\author{
Diktys Stratakis and H. Kamal Sayed \\ Brookhaven National Laboratory, Upton, New York 11973, USA \\ Chris T. Rogers \\ STFC Rutherford Appleton Laboratory, Didcot, OX11 OQX, United Kingdom \\ Androula Alekou \\ CERN, CH-1211 Geneva 23, Switzerland \\ Jaroslaw Pasternak \\ Imperial College, London, SW7 2BW United Kingdom and STFC Rutherford Appleton Laboratory, \\ Didcot, OX11 OQX, United Kingdom
}

(Received 13 October 2013; published 14 July 2014)

\begin{abstract}
An ionization cooling channel is a tightly spaced lattice containing absorbers for reducing the momentum of the muon beam, rf cavities for restoring the longitudinal momentum, and strong solenoids for focusing. Such a lattice can be an essential feature for fundamental high-energy physics applications. In this paper we design, simulate, and compare four individual cooling schemes that rely on ionization cooling. We establish a scaling characterizing the impact of of gradient limitations on the overall performance and systematically compare important lattice parameters such as the required magnetic fields and the number of cavities and absorber lengths for each cooling scenario. We discuss approaches for reducing the peak magnetic field inside the rf cavities by either increasing the lattice cell length or adopting a novel bucked-coil configuration. We numerically examine the performance of our proposed channels with two independent codes that fully incorporate all basic particle-matter-interaction physical processes.
\end{abstract}

DOI: 10.1103/PhysRevSTAB.17.071001

PACS numbers: 29.20.Ej, 41.75.Lx

\section{INTRODUCTION}

Muons are charged particles with mass between those of the electron and proton and can be produced indirectly through pion decay by interaction of a particle beam with a target [1]. Beams of accelerated muons are potentially of great interest for fundamental research as well as for various industrial applications. For instance, accelerated muon beams can enable unique element analysis via muonic $\mathrm{x}$ rays and muon radiography [2]. In addition, compact muon accelerators are desired for medical [3] and material detection applications [4]. Moreover, muon accelerators are being explored for a Neutrino Factory [5] and a Muon Collider [6]. Unfortunately, most of the created muons have diffuse energies and are spread in all directions from the target [7]. Thus, the common task is to capture a large fraction of a divergent muon beam from a production target and cool it promptly so that it will fit within the acceptance of downstream accelerators.

Published by the American Physical Society under the terms of the Creative Commons Attribution 3.0 License. Further distribution of this work must maintain attribution to the author(s) and the published article's title, journal citation, and DOI.
Given the short muon lifetime, ionization cooling is the only practical cooling method that can be realized $[8,9]$. In ionization cooling, the beam loses both transverse and longitudinal momentum as it passes through a material medium. Subsequently, the longitudinal momentum can be restored by reacceleration, leaving a net loss of transverse momentum. For muon accelerator applications, this transverse cooling is achieved in a series of cells. Each cell consists of solenoids for focusing, disk-shaped absorbers where cooling takes place, and rf cavities to replenish the energy lost in the absorbers. Another variant of this technique is to replace the disc absorbers with a continuous absorber [10] wherein all of the cooling section, including the rf cavities, is filled with dense hydrogen gas [11,12].

It is important to emphasize that vacuum rf cooling lattices designed so far require normal conducting $201-805 \mathrm{MHz}$ cavities to operate within strong magnetic fields [13-16]. For instance, nominal rf gradients in the Neutrino Factory baseline cooling channel are $16 \mathrm{MV} / \mathrm{m}$ at $201 \mathrm{MHz}$, while the magnetic field alternates between $-3 \mathrm{~T}$ and $3 \mathrm{~T}$ [16]. Experimental [17] and numerical [18] studies have indicated that the vacuum rf gradient may be limited by the magnetic field, and it is uncertain whether the gradients specified for the cooling sections can be achieved. For this reason it is important to examine possible mitigation techniques, so that 
we can avoid any rf voltage suppression due to the presence of strong magnetic fields [19].

In this paper we discuss in more detail novel lattice designs that allow the operation of an rf cavity within subtesla magnetic fields. We numerically examine the performance of four individual cooling lattices by constructing a complete simulation model with two independent codes that fully incorporate all basic physical processes such as energy loss, scattering, straggling, and muon decay. Then, we evaluate the different lattices by systematically comparing various lattice parameters such as the required magnetic fields, the transverse beta functions and the absorber lengths for each specific case.

Our studies indicate the sensitivity of the channel performance to variations in the design parameters. For instance, we numerically study the impact of rf gradient limitations on the overall performance for each ionization cooling scheme. We show that by using a novel radial bucked coil (RBC) [20] configuration we can reduce the magnetic field on the cavity iris by a factor of 3 , while at the same time we obtain a muon yield that is only $5 \%$ lower than that of the baseline cooling channel.

The layout of this paper is as follows: In Sec. II, we give an overview of the ionization cooling concept. In Sec. III we provide details of the design parameters for our proposed cooling channels. Then, with the aid of two independent codes we demonstrate the cooling efficacy of each lattice (Sec. IV). Finally, we present our conclusions in Sec. V.

\section{COOLING IN PARTICLE-MATTER- INTERACTION SYTEMS}

Ionization cooling involves passing the beam through some material in which the muons lose both transverse and longitudinal momentum by ionization energy loss, commonly referred to as $d E / d x$. The longitudinal momentum can be restored by reacceleration, leaving a net loss of transverse momentum. The equation describing transverse ionization cooling is a balance between cooling (first term) and heating (second term) effects [21]:

$$
\frac{\mathrm{d} \varepsilon_{\mathrm{n}}}{\mathrm{ds}} \approx-\frac{1}{\beta^{2}} \frac{\mathrm{dE}_{\mu}}{\mathrm{ds}} \frac{\varepsilon_{\mathrm{n}}}{\mathrm{E}_{\mu}}+\frac{1}{\beta^{3}} \frac{\beta_{\mathrm{T}} \mathrm{E}_{\mathrm{S}}^{2}}{2 \mathrm{E}_{\mu} \mathrm{m}_{\mu} \mathrm{c}^{2} \mathrm{~L}_{\mathrm{R}}},
$$

where $\varepsilon_{n}$ is the normalized transverse emittance, $E_{\mu}$ is the muon energy, $m_{\mu}$ is the muon mass, $\beta_{T}$ is the transverse betatron function at a discrete absorber, $\beta$ is the relativistic beta, $c$ is the speed of light, $d E_{\mu} / d s$ is the energy loss per unit length, $L_{R}$ is the radiation length of the material, and $E_{s}$ is the characteristic scattering energy $(\sim 13.6 \mathrm{MeV})$.

The minimum normalized transverse emittance (known as equilibrium emittance) that can be achieved for a given absorber in a given focusing field is reached when the cooling term equals the heating term in Eq. (1):

$$
\varepsilon_{n, \min } \approx \frac{\beta_{T} E_{s}^{2}}{2 \beta m_{\mu} c^{2} L_{R}\left|\frac{d E_{\mu}}{d s}\right|} .
$$

One wants to use absorber materials for which the product of radiation length and energy loss is large. Hydrogen and lithium hydride $(\mathrm{LiH})$ are the most common choices. Another parameter that can be controlled is the beta function, which we want to keep as small as possible over the length of the absorber.

Transverse ionization cooling can take place in principle at any momentum. However, at low momentum the slope of the $d E / d x$ curve causes lower momentum particles to lose more energy than higher energy particles. This increases the energy spread and leads to a blowup in the longitudinal emittance. Cooling at high momentum is uneconomical since a lot of $\mathrm{rf}$ power is required to replace a fixed fraction of the initial energy. For these reasons cooling channels are typically designed with a reference momentum near the minimum of the $\mathrm{dE} / \mathrm{dx}$ curve $(\approx 200 \mathrm{MeV} / c)$ [22].

For the Neutrino Factory, the transverse emittance of the muon beam must be reduced in order to fit into the downstream accelerators and storage ring. Ionization cooling is achieved in a series of cells [23] that (1) lower the beam energy by $\sim 10 \mathrm{MeV}$ in Lithium Hydride $(\mathrm{LiH})$ absorbers, (2) use $201 \mathrm{MHz}$ rf cavities to restore the lost energy, and (3) use solenoids with $\sim 3 \mathrm{~T}$ peak field on-axis to strongly focus the beam at the absorbers. Each lattice cell contains one solenoid and the direction of the solenoidal field reverses with every cell repetition in order to prevent the buildup of canonical angular momentum. The lattice efficiency can be evaluated by calculating the muon yield, which is defined as the number of particles that fall within a reference acceptance, which approximates the expected acceptance of the downstream accelerator. For the Neutrino Factory case, the transverse normalized acceptance is $30 \mathrm{~mm}$ and the normalized longitudinal acceptance is $150 \mathrm{~mm}$ [16]. At the end of the cooling channel the rms normalized emittance is expected to drop by a factor of 3 , while the accepted muon yield will rise by a factor of $\sim 2$.

As mentioned in the Introduction, technical risks to the existing cooling channel designs are presented by the operation of rf cavities in the presence of strong magnetic fields. Three experiments at the Fermilab MuCool Test Area [17] studied the effect of the external magnetic field on the breakdown behavior of cavities. The first experiment had a single pillbox [17], the second had a box cavity $[24,25]$, and the third an all-season cavity [26,27]. The allseason refers to a modular pillbox with replaceable end walls designed for both vacuum and high-pressure experiments. In all experiments, operating the rf cavity in a magnetic field caused either a decline in the achievable gradient or surface damage, or both. For this reason, it seems prudent to begin investigating new cooling schemes that not only manage to reduce significantly the muon transverse emittance and obtain adequate muon 


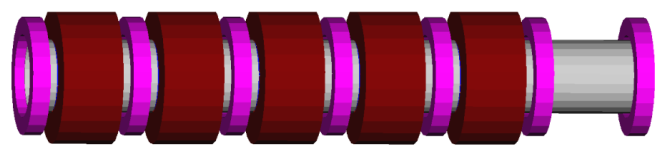

(a)

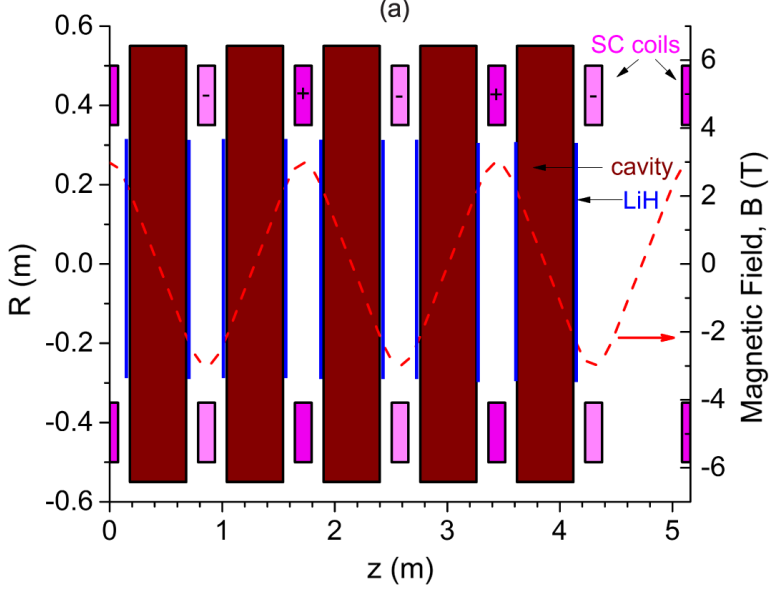

(b)

FIG. 1. Schematic representation of the baseline lattice: (a) Configuration in 3D; (b) side view. Note that six cells are shown. The magenta cylinders are solenoids, the red cylinders are the active volume of the rf cavities, and the blue blocks are lithium hydride $(\mathrm{LiH})$ absorbers. The dashed red line shows the magnetic field along the axis. The current density of each coil is $105.6 \mathrm{~A} / \mathrm{mm}^{2}$. transmission, but that also achieve a small magnetic field at the rf cavity wall.

\section{ALTERNATIVE LATTICE DESIGNS FOR MUON COOLING}

Figure 1 displays a representative portion of the cooling channel that is currently considered to be the baseline (BASE) for the Neutrino Factory Front-End cooling scenario. The channel consists of a sequence of identical cells, each containing one $50 \mathrm{~cm}$ long pillbox cavity and two thick disk-shaped absorbers that cause energy loss. This configuration is a revised version of the IDS-NF baseline published in Ref. [28] with the only difference being that the two adjacent $25 \mathrm{~cm}$ long $201.25 \mathrm{MHz}$ cavities were replaced with a single cavity since this would represent a more realistic configuration. Each cell contains one solenoid coil, the polarity of which flips from cell to cell, yielding an approximately sinusoidal variation of the magnetic field with a peak value of $\sim 3 \mathrm{~T}$ on axis (red dashed curve). The axial length of the solenoid is $15 \mathrm{~cm}$, with an inner radius of $35 \mathrm{~cm}$, an outer radius of $50 \mathrm{~cm}$, and a current density of $105.6 \mathrm{~A} / \mathrm{mm}^{2}$. Six cells of the channel are displayed in Fig. 1 and more information can be found in Tables I and II. In order to allow sufficient space between cavities and coils and to allow easier removal of parts of the lattice the cell length is set to $86 \mathrm{~cm}$ and an empty cell is

TABLE I. Lattice parameters of four alternative muon ionization cooling scenarios. Transverse beta functions are calculated at the reference momentum.

\begin{tabular}{|c|c|c|c|c|}
\hline & BASE & $\mathrm{RBC}$ & LBC & ICL \\
\hline Reference momentum $(\mathrm{MeV} / c)$ & 230 & 230 & 230 & 230 \\
\hline Cell length $(\mathrm{cm})$ & 86 & 105 & 86 & 300 \\
\hline $\mathrm{rf}(\mathrm{MHz})$ & 201.25 & 201.25 & 201.25 & 201.25 \\
\hline Absorber type & $\mathrm{LiH}$ & $\mathrm{LiH}$ & $\mathrm{LiH}$ & $\mathrm{LiH}$ \\
\hline Absorber length per cell $(\mathrm{cm})$ & 1.8 & 1.9 & 2.2 & 4.4 \\
\hline rf phase (deg.) & 35 & 43 & 43 & 30 \\
\hline Minimum beta function $(\mathrm{cm})$ & 72 & 85 & 95 & 89 \\
\hline Beta function at absorber center $(\mathrm{cm})$ & 79 & 92 & 101 & 89 \\
\hline Equilibrium emittance (mm) & 5.0 & 5.8 & 6.4 & 5.6 \\
\hline Length of cooling channel (m) & 148 & 148 & 100 & 183 \\
\hline Number of cavities & 143 & 117 & 97 & 122 \\
\hline rf gradient $(\mathrm{MV} / \mathrm{m})$ & 16.0 & 16.0 & 16.0 & 16.0 \\
\hline rf length $(\mathrm{cm})$ & 50 & 50 & 50 & 50 \\
\hline Total rf length/total absorber length & 23.0 & 22.0 & 19.0 & 22.7 \\
\hline Muon yield & 1.97 & 1.86 & 1.76 & 1.55 \\
\hline Final trans. emittance (mm) & 6.5 & 6.5 & 6.7 & 7.0 \\
\hline
\end{tabular}

TABLE II. Lattice characteristics of each cooling scenario.

\begin{tabular}{lcccc}
\hline \hline & BASE & RBC & LBC & ICL \\
\hline Current density $\left(\mathrm{A} / \mathrm{mm}^{2}\right)$ & 105.6 & $120.0 / 90.0$ & $167.0 / 200.0$ & 19.3 \\
Maximum total field on coil (T) & 7.2 & 6.9 & 12.2 & 2.0 \\
Maximum total field on axis (T) & 3.0 & 2.8 & 2.8 & 1.8 \\
Maximum hoop stress $(\mathrm{MPa})$ & 270 & 340 & 470 & 15 \\
\hline \hline
\end{tabular}




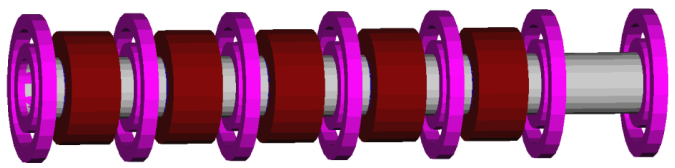

(a)

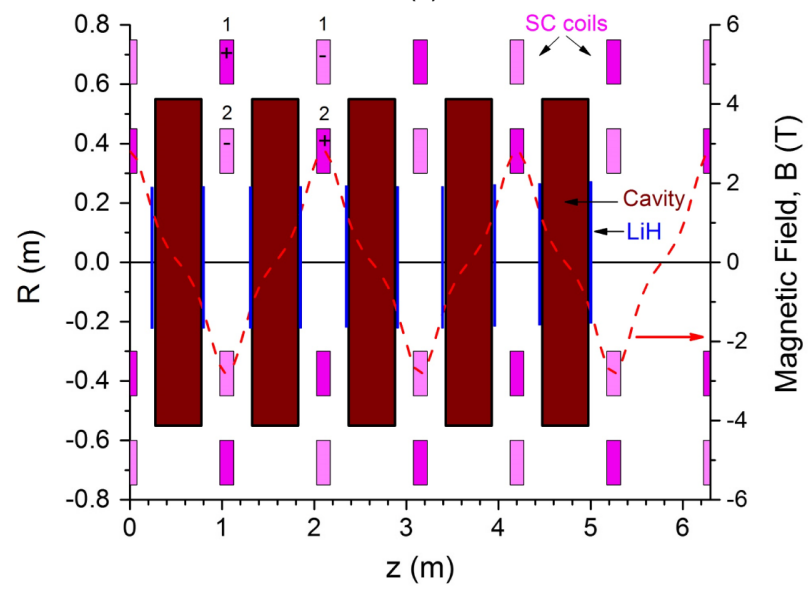

(b)

FIG. 2. Schematic representation of the RBC lattice: (a) Configuration in $3 \mathrm{D}$; (b) side view. Note that six cells are shown. The magenta cylinders are solenoids, the red cylinders are the active volume of the rf cavities, and the blue blocks are $\mathrm{LiH}$ absorbers. The dashed red line shows the magnetic field along the axis. The current densities for coils 1 and 2 are 90 and $120 \mathrm{~A} / \mathrm{mm}^{2}$, respectively.

added after five cavities. The same pattern is repeated as necessary until the desired emittance reduction is achieved.

The cavities have a frequency of $201.25 \mathrm{MHz}$ and a nominal gradient of $16 \mathrm{MV} / \mathrm{m}$. The cooling channel was designed to have a transverse beta function that is relatively constant with position and this allows the use of the rf cavity windows as the cooling absorbers. The window consists of a $0.9 \mathrm{~cm}$ thick $\mathrm{LiH}$ absorber with a $100 \mu \mathrm{m}$ thick layer of beryllium $(\mathrm{Be})$ on the side facing the cavity, and a $25 \mu \mathrm{m}$ thick layer of Be on the opposite side.

Next, we explore lattices with shielded cavities where the magnetic field within the cavity is significantly lower. Two lattices are examined, one with shielding provided by bucked coils [29] arranged radially (Fig. 2) and one with shielding provided by bucked coils arranged longitudinally (Fig. 3).

Figure 2 displays the layout of six cells of a RBC scheme. Notice that each lattice cell consists of the same components as the BASE cell but has a longer length and uses a pair of bucked coils rather than a single coil. The two coils have different radii, opposite polarities, and are placed at the same position along the beam axis. Like in the BASE, the polarity of each coil flips from cell to cell.

Each RBC lattice cell is $105 \mathrm{~cm}$ long and contains two $0.95 \mathrm{~cm}$ thick disk-shaped LiH absorbers and a $50 \mathrm{~cm}$ long 201.25 MHz cavity. The axial magnetic field peaks at $2.8 \mathrm{~T}$ and provides transverse focusing with $\beta_{T} \approx 85 \mathrm{~cm}$. The

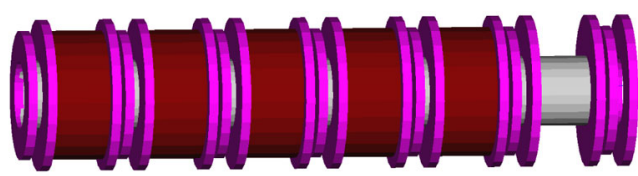

(a)

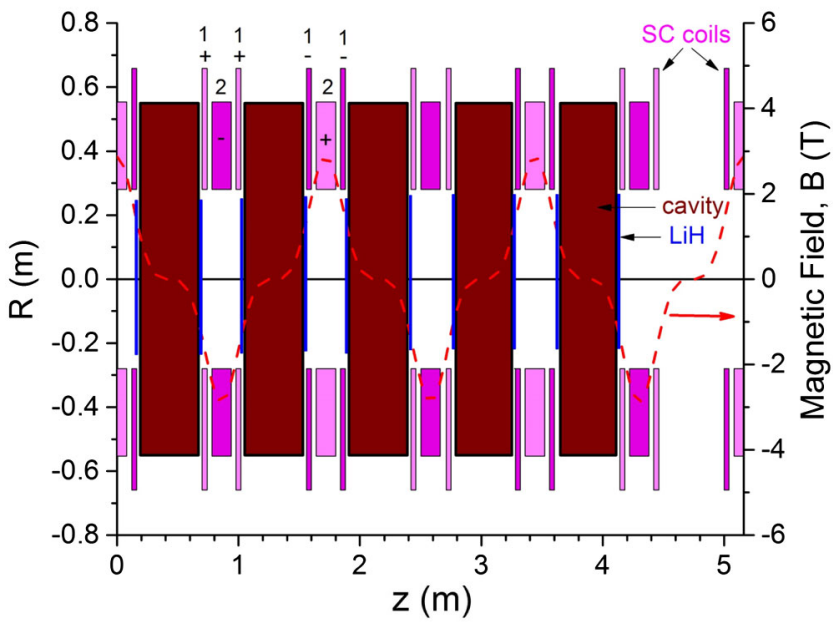

(b)

FIG. 3. Schematic representation of the LBC lattice: (a) Configuration in 3D; (b) side view. Note that six cells are shown. The magenta cylinders are solenoids, the red cylinders are the active volume of the rf cavities, and the blue blocks are $\mathrm{LiH}$ absorbers. The dashed red line shows the magnetic field distribution along the axis. The current densities for coils 1 and 2 are 200 and $167 \mathrm{~A} / \mathrm{mm}^{2}$, respectively.

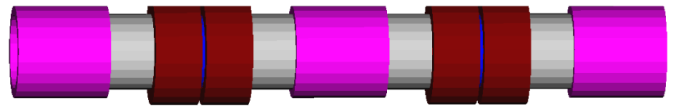

(a)

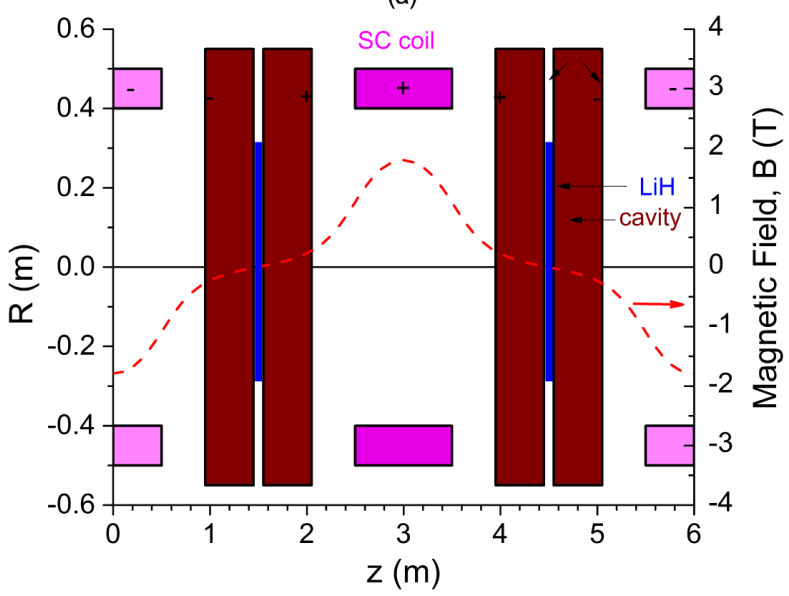

(b)

FIG. 4. Schematic representation of the ICL lattice: (a) Configuration in 3D; (b) side view. Note that two cells are shown. The magenta cylinders are solenoids, the red cylinders are the active volume of the rf cavities, and the blue blocks are $\mathrm{LiH}$ absorbers. The dashed red line shows the magnetic field distribution along the axis. The current density of each coil is $19.3 \mathrm{~A} / \mathrm{mm}^{2}$. 
configuration of the coils leads to a notable reduction of the magnetic field within and near the rf cavity (red dashed curve). Quantitatively, the axial magnetic field at the cavity wall is just $1.0 \mathrm{~T}$, which is a factor of 2 less than the BASE field. One can see from Fig. 3 that a very similar drop of the magnetic field near the cavity region can be achieved by using a longitudinal bucked coil (LBC) scheme. The only difference is that the cell length is now reduced to $86 \mathrm{~cm}$.

From the results in Table II it becomes clear that, unlike the BASE and RBC lattices, the maximum field on the coil for the LBC scheme is close to the published engineering limits [30] for NbTi based solenoids. In addition, the coils are placed very close to each other, causing the maximum hoop stress, $\sigma_{t}$, to be $470 \mathrm{MPa}$, which is notably higher compared to the BASE and RBC configurations. On the promising side, this value is below the reported [31] working maximum hoop stress for $\mathrm{NbTi}$ composites, which is $500 \mathrm{MPa}$. Note that for our estimates we use the "current sheet approximation" [32] where the current flows in a thin surface around the coil circumference. The approximate hoop stress acting on a solenoid is $\sigma_{t}=J_{t} B_{z} r$, where $J_{t}$ is the current density, $B_{z}$ is the longitudinal magnetic field component, and $r$ is the radius.

Another option for ionization cooling is to modify the BASE channel by increasing its cell-length so that the rf cavities no longer sit in intense magnetic fields. A key advantage of this method is that the technical risk associated with the design is greatly reduced compared to the BASE since the maximum hoop stress drops to values that are below $20 \mathrm{MPa}$. On the downside, as predicted in Ref. [33] and confirmed by simulations presented below, the increased cell length leads to either weaker focusing and worse cooling performance, or decreased acceptance and worse transmission.

Figure 4 shows the layout of two cells of a cooling channel with an increased cell length (ICL) configuration. More information about the lattice parameters can be found in Tables I and II. Notice that now the length of each cell is increased to $300 \mathrm{~cm}$, which is a factor of 3.5 longer than the BASE lattice cell. Each cell contains two $50 \mathrm{~cm}$ long rf cavities which are separated by a $4.4 \mathrm{~cm}$ long $\mathrm{LiH}$ absorber. The lattice consists of identical solenoids with $200 \mathrm{~cm}$

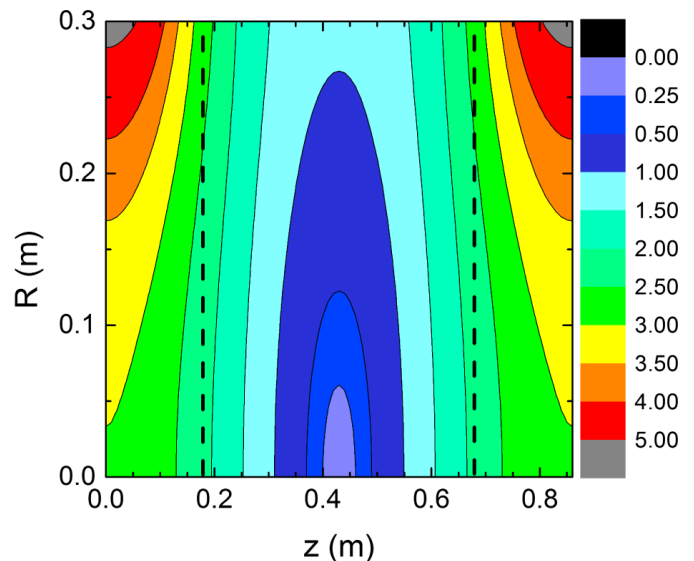

(a)

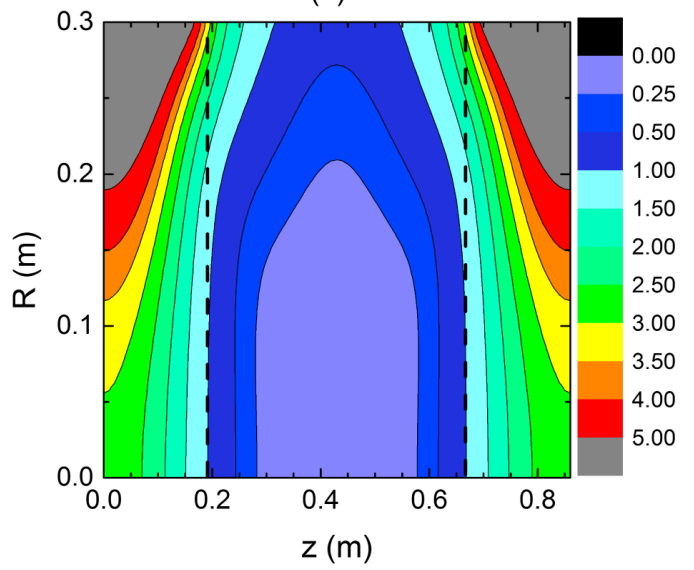

(c)

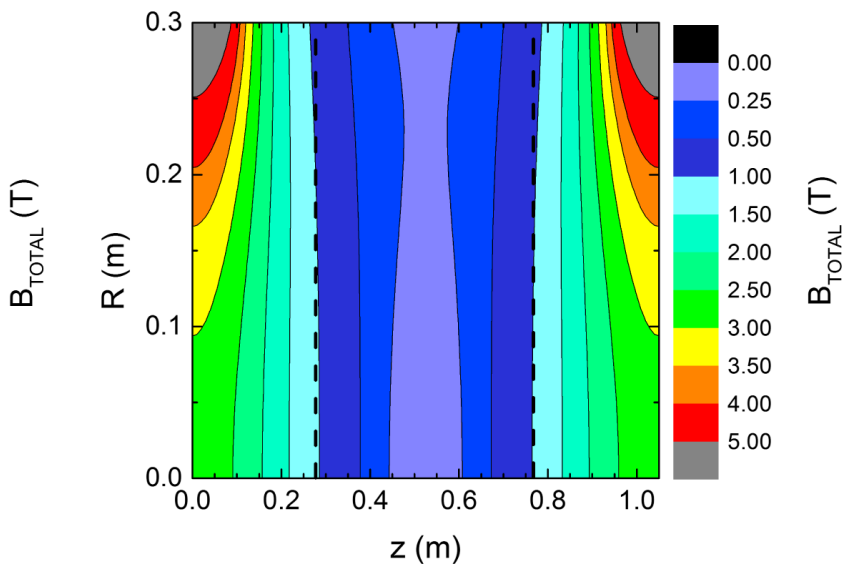

(b)

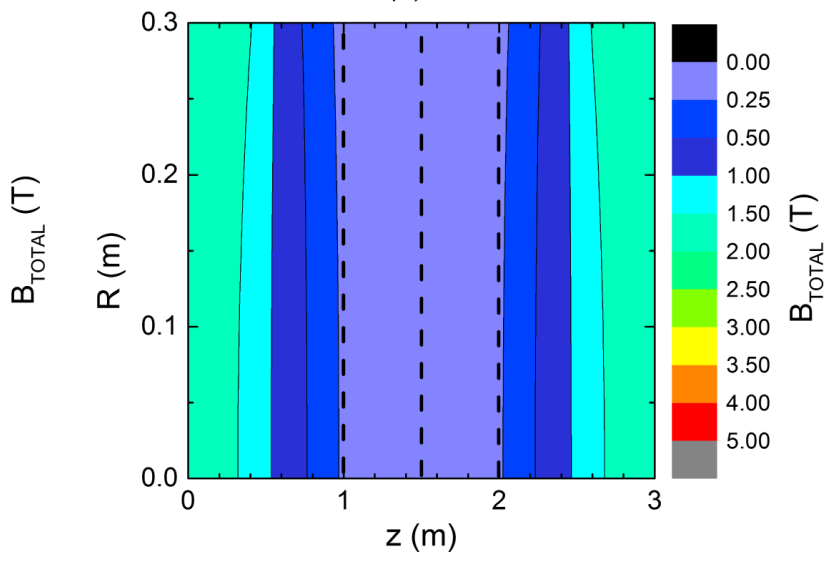

(d)

FIG. 5. Color map plot of the total magnetic distribution, $\mathrm{B}_{\mathrm{TOTAL}}$ (in T) versus $z$ along one cell for (a) BASE lattice; (b) RBC lattice; (c) LCB lattice, and (d) ICL lattice. Dashed line indicates the position of the wall of the rf cavity. 


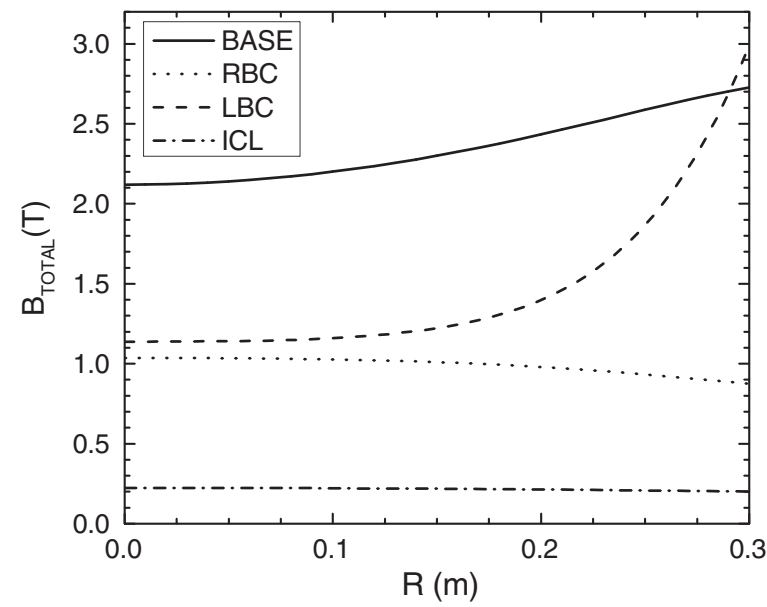

FIG. 6. Total magnetic field with respect to the radius, $R$, for the $z$ position corresponding to the wall of the rf cavity.

separation, with adjacent coils having opposite polarities. This yields a sinusoidal variation of the axial magnetic field in the channel with a peak value of $1.8 \mathrm{~T}$, providing a transverse beta function smoothly varying along each cell, with a minimum value of $89 \mathrm{~cm}$. The axial length of the coil is $100 \mathrm{~cm}$, with an inner radius of $40 \mathrm{~cm}$, an outer radius of $50 \mathrm{~cm}$, and a current density of $19.3 \mathrm{~A} / \mathrm{mm}^{2}$. The low current density relative to that of the BASE lattice is seen as an advantage, as it may enable a more conservative temperature margin to be used in a linac that may have significant losses.

Previously conducted numerical studies [18] have shown that when the magnetic field exceeds $1 \mathrm{~T}$, fieldemitted electrons can deposit enough energy on the cavity surface to create damage and initiate breakdown. Consequently, the primary goal of our study is to implement a new lattice design so that the rf cavities are placed in a region with total magnetic field $B_{\text {TOTAL }} \leq 1 \mathrm{~T}$. To

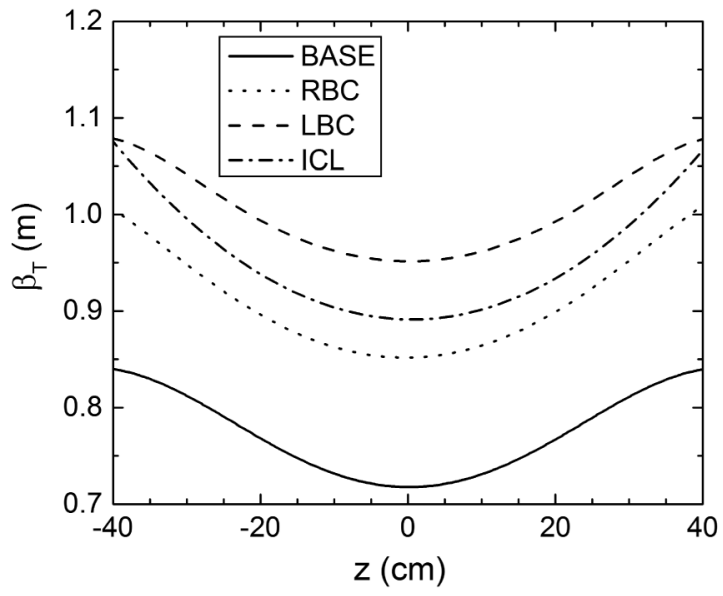

(a) examine this, in Fig. 5 we show the total magnetic field profile along the cell of each of the aforementioned lattices, from the coil center to $30 \mathrm{~cm}$ in radius. A common feature of all lattices is that the magnetic field is zero at the cell-center since the polarity of the coils flips in the middle of the cell with every coil repetition. However, there is a noticeable difference in the field strength within the cavity region. For instance, the area with $\mathrm{B}_{\text {TOTAL }}<0.5 \mathrm{~T}$ extends to less than $12 \mathrm{~cm}$ radius at the center of the cavity for the BASE, while for both RBC and ICL schemes it exceeds $30 \mathrm{~cm}$. In the longitudinal direction, in the case of the LBC lattice, more than $60 \%$ of the cavity sits in a region where $\mathrm{B}_{\text {TOTAL }}<0.25 \mathrm{~T}$, while for both RBC and ICL schemes the whole cavity is positioned in an area where the total field is $\leq 1 \mathrm{~T}$.

Figure 6 displays the total magnetic field versus radius for the $z$ position corresponding to the wall of the cavity. This position has been chosen since the magnetic field strength at the cavity wall, and particularly the iris $(\mathrm{R}=0.3 \mathrm{~m})$, has been long considered to be the main parameter which limits the accelerating gradient, because of its direct role in field emission [18,19]. Note that the magnetic field at the iris for the BASE lattice cell is 3 and 12 times greater compared to the RBC and ICL schemes, respectively. In addition, the magnetic field at the edge of the rf cavity for the ICL and RBC schemes is $\leq 0.25 \mathrm{~T}$ and $\leq 1 \mathrm{~T}$ for any radius, respectively. The fact that the field on the wall rises steadily for $\mathrm{R}>0.15 \mathrm{~m}$ for the LBC scheme, in combination with the engineering constraints discussed earlier, makes this lattice the most challenging option.

Figure 7 shows the transverse betatron function, $\beta_{T}$, versus axial position [Fig. 7(a)] and momentum [Fig. 7(b)]. Note that $z=0 \mathrm{~cm}$ corresponds to the center of the lattice cell for all cases. Clearly, $\beta_{T}$ becomes minimum at the cell center for all scenarios and since the lattice equilibrium emittance is proportional to the beta

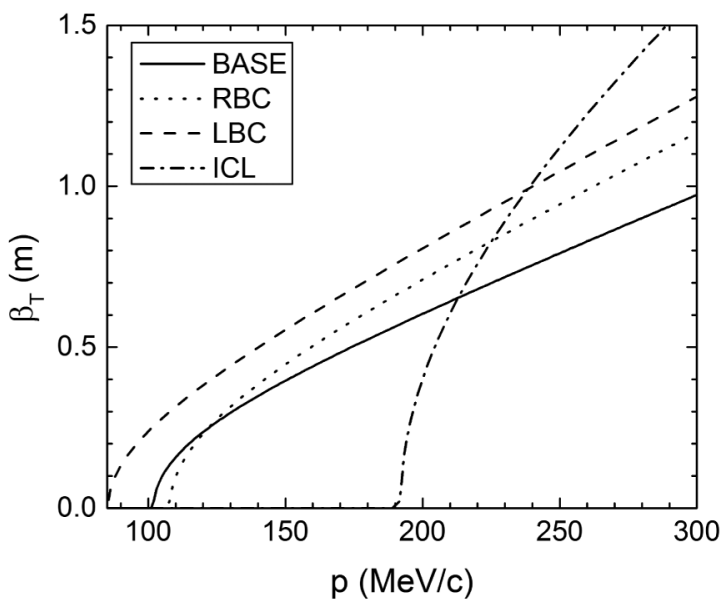

(b)

FIG. 7. Evaluation of lattice functions for the different cooling schemes: (a) Transverse betatron function along the beam axis at $230 \mathrm{MeV} / c$; and (b) transverse betatron function with respect to the total momentum. The cell center is at $z=0$. 
function at the absorber, ideally one would like to place the absorber at this location. While the absorber is located at the cell center in the ICL scheme, in the other lattices it is offset by $25 \mathrm{~cm}$ (from the center) due to the placement of the rf cavity. Nevertheless, the beta value at the absorber is still low enough to provide an equilibrium emittance that is below $7.0 \mathrm{~mm}$ for all cases (see Table I), which meets the Neutrino Factory cooling criterion [16]. Figure 7(b) shows the dependence of beta on momentum for the BASE, RBC, LBC, and ICL schemes. The BASE and both bucked-coil schemes have better acceptance compared to the ICL since the range of momentum over which beta is nonzero is wider.

\section{PERFORMANCE OF LATTICES}

The performance of the cooling channels was simulated using both the ICOOL [34] and G4beamline codes [35]. Both are standard codes for the Muon Accelerator Program (MAP) [36] that tracks all relevant physical processes (e.g. energy loss, straggling, multiple scattering) and include muon decays. For each cell, we generated 2D cylindrical field maps by superimposing the fields from all solenoids in the cell and its neighboring cells. The resultant field components were shown to satisfy Maxwell's equations to a high level of accuracy and agreed with independent calculations. The rf cavities were modeled using cylindrical pillboxes running in the TM010 mode and a reference particle was used to determine each cavity's relative phase. Both irises were covered by a thin Be window in order to produce the maximum electric field on axis for a given amount of rf power [37]. The absorber material was $\mathrm{LiH}$, which was enclosed in Be safety windows. For simplicity, we assumed that the windows are planar and located axially on both sides of the absorber.

The input beam in the simulations has a normalized transverse emittance of $17.0 \mathrm{~mm}$, a normalized longitudinal emittance of $50.0 \mathrm{~mm}$, and a reference momentum equal to $230 \mathrm{MeV} / c$. These parameters closely resemble the distribution of a muon beam after it exits the phase-rotation section of the Neutrino Factory Front-End [28]. The normalized emittance has been obtained using ECALC9 $\mathrm{f}$ [38], an emittance calculation program customarily employed by MAP. In the calculation, a factor $1 / m_{\mu} c$ was introduced so as to express the longitudinal emittance in units of length. A histogram of the momentum distribution at the beginning of the channel is shown in Fig. 8.

Next, using both ICOOL and G4beamline we examine the performance of our cooling schemes as a function of channel length. We tracked 40,000 particles and included muon decay. Our main findings are summarized in Table I and are examined in more detail in the remainder of this section. We evaluated the cooling performance by counting the number of accepted particles (muon yield) that fall within a transverse normalized acceptance of $30 \mathrm{~mm}$ and a normalized longitudinal acceptance of $150 \mathrm{~mm}$. For all

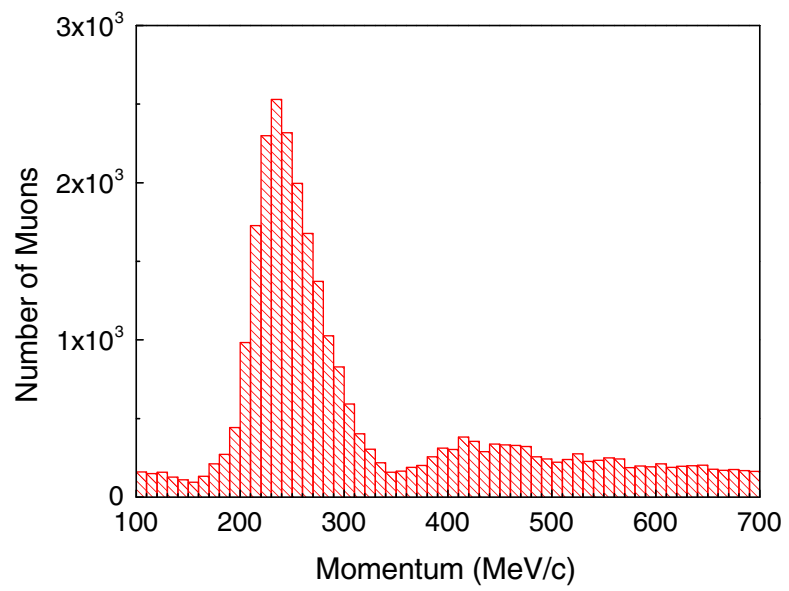

FIG. 8. Histogram of the momentum distribution of the beam at the entrance of the channel.

cooling scenarios examined, we performed one-dimensional scans wherein we varied key parameters such as the rf phase and absorber thickness in order to find the peak muon yield for each variable. For instance, in Fig. 9 we show the sensitivity in performance as we vary the length of the absorber. The rf phase and absorber length that provide the peak muon yield for each cooling scheme are listed in Table I. Note that we found the same yield by using the conventional algorithm "Nelder-Mead" $[39,40]$ by scanning both rf phase and absorber length.

Figure 10(a) shows the number of accepted muons that fit within the above transverse and longitudinal acceptances as a function of longitudinal position for the optimum absorber length derived from Fig. 9. The solid curve is with the muon decay enabled in the simulation and the dashed curve is with muon decay disabled. Note that for all cases a nominal $\mathrm{rf}$ gradient of $16 \mathrm{MV} / \mathrm{m}$ is assumed and a yield equal to one corresponds to the yield at the cooler entrance. Clearly, the BASE lattice achieves the highest muon yield which peaks at 1.97 according to ICOOL and 1.98 according to G4beamline, at $z=148 \mathrm{~m}$. Not far from this value is the performance of the RBC lattice. In particular, ICOOL finds the peak muon yield for this lattice to be 1.86 and G4beamline 1.87, which is only 5\% less than that of the BASE lattice. It is important to emphasize that the total cooling length that is required to achieve the peak muon yield is the same for both BASE and RBC schemes. Since the $\mathrm{RBC}$ cell is longer than the BASE cell, an implication of this result is that the $\mathrm{RBC}$ scheme would require $20 \%$ fewer cavities, making it a potentially cost-effective option [41]. On the other hand, it will require twice as many coils per cell and the added coils are likely to be technically more challenging compared to the BASE channel. The LBC channel seems also an attractive option since its peak performance is at $z=100 \mathrm{~m}$ making this channel the shortest among all. On the downside, its yield is $11 \%$ lower (1.76 in ICOOL and G4beamline) compared to that 


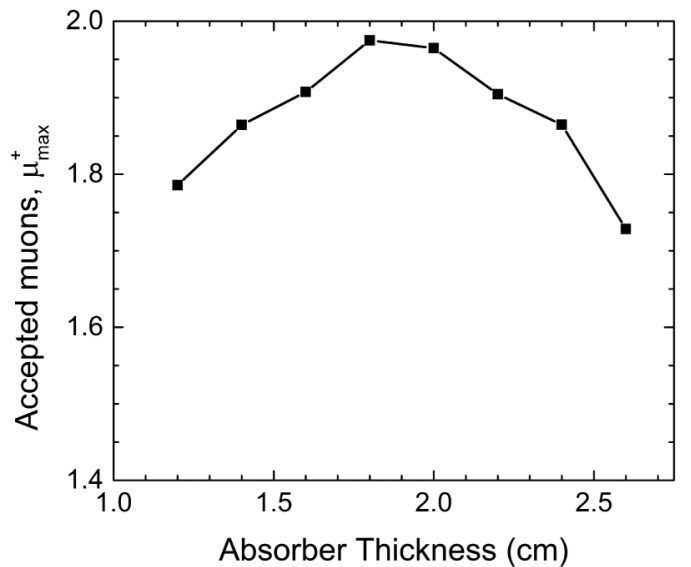

(a)

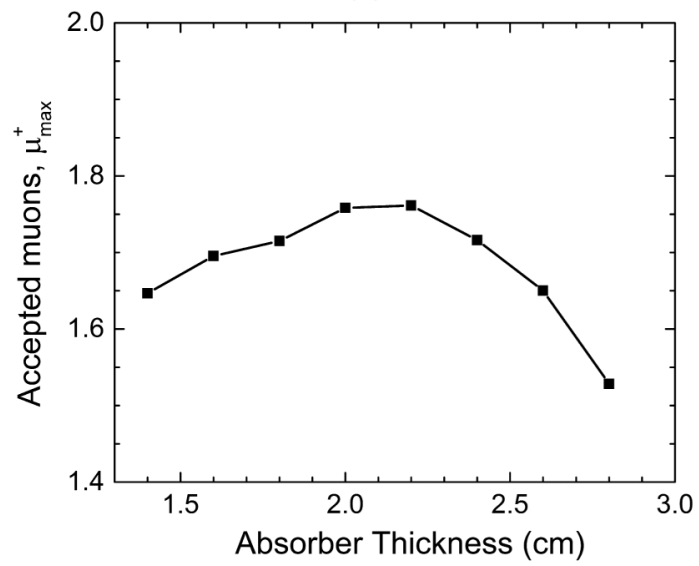

(c)

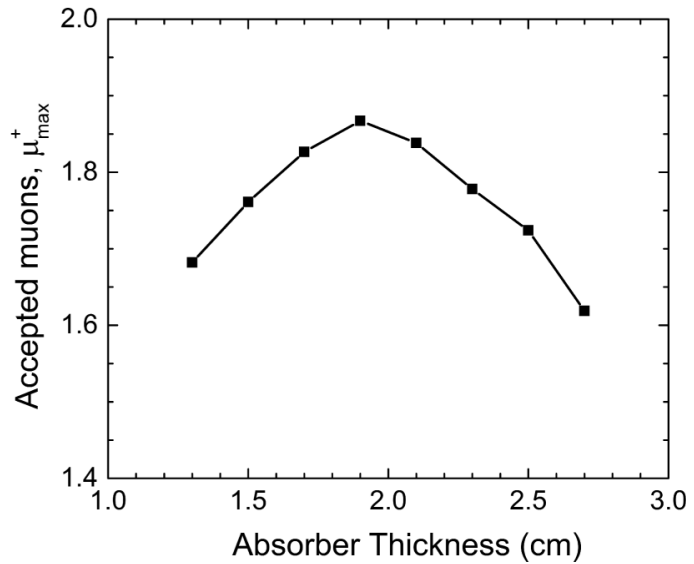

(b)

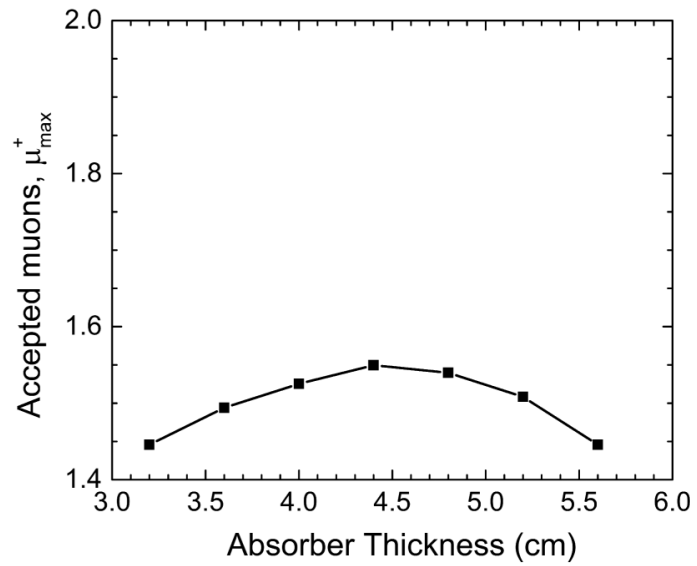

(d)

FIG. 9. ICOOL simulation results of the accepted muon yield for different absorber lengths per cell: (a) BASE, (b) RBC, (c) LBC, and (d) ICL schemes.

of the BASE lattice. While the ICL lattice has the lowest peak yield compared to the other schemes, which is 1.55 in ICOOL (1.50 in G4beamline) at $z=183 \mathrm{~m}$, this scheme has also the lowest magnetic field on the rf cavity wall, which is $\leq 0.25 \mathrm{~T}$.

Figure 10(b) examines the normalized transverse emittance reduction as a function of distance along the channel. The BASE channel produces a final value of $6.5 \mathrm{~mm}$ at $z=148 \mathrm{~m}$, i.e., almost a factor of 3 smaller than its initial value. A very similar performance is obtained by the $\mathrm{RBC}$ lattice. The bumps near the start of the LBC lattice are most likely from particle losses arising from a slight mismatch as the beam propagates from the phase rotator to the cooling system. At $z=100 \mathrm{~m}$, it cools to an emittance of $6.7 \mathrm{~mm}$ which resembles closely the performance of the other lattices. Finally, the predicted emittance for the ICL at $z=$ $183 \mathrm{~m}$ is near $7.0 \mathrm{~mm}$. Note from Fig. 7(b) that the ICL lattice accepts particles with a momentum greater than $190 \mathrm{MeV} / c$. On the other hand, it is clear from Fig. 8 that there are a considerable number of particles $(\sim 8 \%)$ with a momentum $\leq 190 \mathrm{MeV} / c$ which means that the initial emittance drop for the ICL is not due to cooling but from particle loss. We note that for all the cooling scenarios we discussed, the final values for the transverse emittance, $\varepsilon_{T}$, meet the Neutrino Factory criterion, which requires $\varepsilon_{T} \leq 7.0 \mathrm{~mm}$. Finally, ICOOL and G4beamline provide results with a high level of agreement for both muon yield and emittance.

In Fig. 11 we attempt to establish a scaling characterizing the impact of rf gradient limitations on the achieved maximum muon yield. As before, a yield equal to one corresponds to the yield at the cooler's entrance. It is clear that the cooling performance strongly depends on the rf voltage. Quantitatively, a decline of gradient from the nominal value $(16 \mathrm{MV} / \mathrm{m})$ to $12 \mathrm{MV} / \mathrm{m}$ in the BASE lattice would cause a drop in performance by more than $15 \%$. While the BASE achieves a 5\% higher muon yield compared to the RBC channel for any rf gradient, results from previously conducted experimental work [17] suggest that the maximum achievable rf gradient is correlated with the value of the magnetic field at the cavity wall and particularly the iris. That means the RBC scheme could 


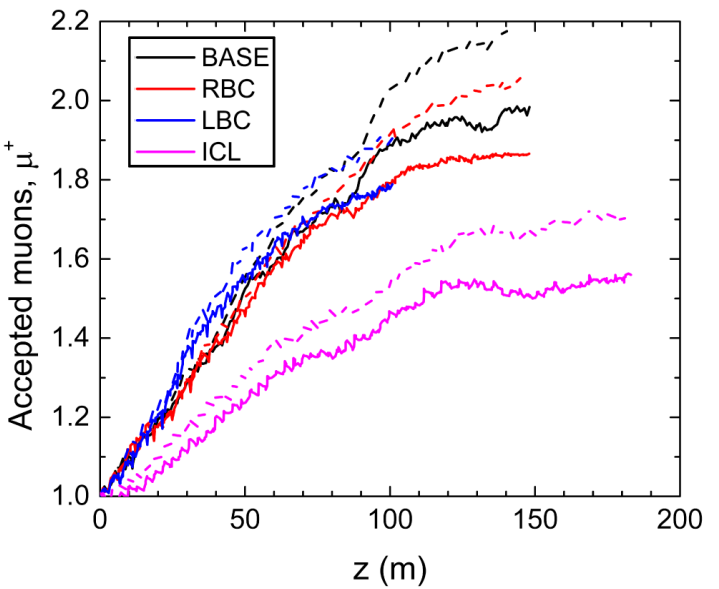

(a)

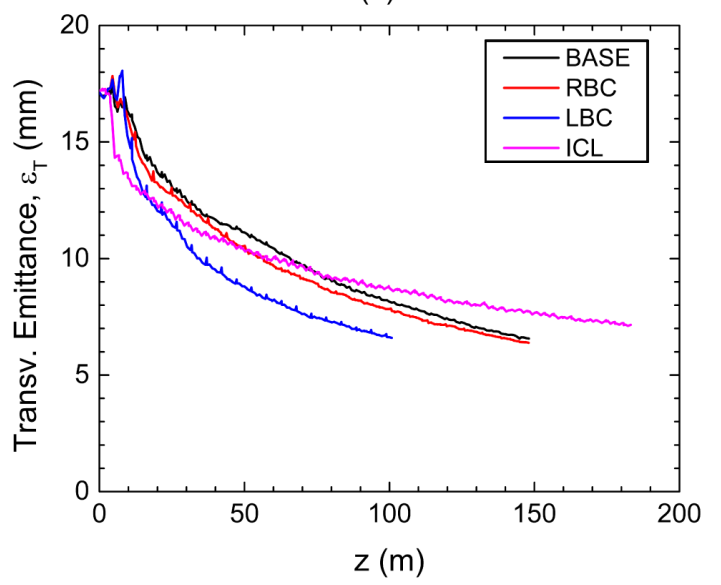

(b)

FIG. 10. ICOOL simulation results of the cooling performance as a function of distance along the channel: (a) The accepted muon yield within a transverse acceptance of $\mathrm{A}_{\mathrm{T}} \leq 30 \mathrm{~mm}$ and longitudinal acceptance of $A_{L} \leq 150 \mathrm{~mm}$. (b) Normalized transverse rms emittance. In (a) the dashed lines are with muon decay off and solid lines are with decay on.

safely operate at higher gradients since the magnetic field on the cavity iris is reduced by a factor of 3 . In that scenario, the RBC can perform as well as the baseline. For instance, the RBC at $19 \mathrm{MV} / \mathrm{m}$ closely matches the BASE at the nominal gradient with a drawback, however, that it requires more rf power. On the other hand, neither the LBC nor the ICL scheme exceeds the BASE muon yield for any voltage.

In conclusion, it becomes evident from Fig. 11 that the performance of any cooling channel largely depends on the gradient that can be achieved within a given magnetic field. In particular, for the Neutrino Factory baseline, it must be proven experimentally that the $201 \mathrm{MHz}$ pillbox rf cavities at $16 \mathrm{MV} / \mathrm{m}$ can operate safely in magnetic fields of up to $3 \mathrm{~T}$. If $16 \mathrm{MV} / \mathrm{m}$ can be sustained only at a $\sim 1 \mathrm{~T}$ magnetic field then the RBC bucked coil scheme looks more appropriate, and according to our numerical estimates

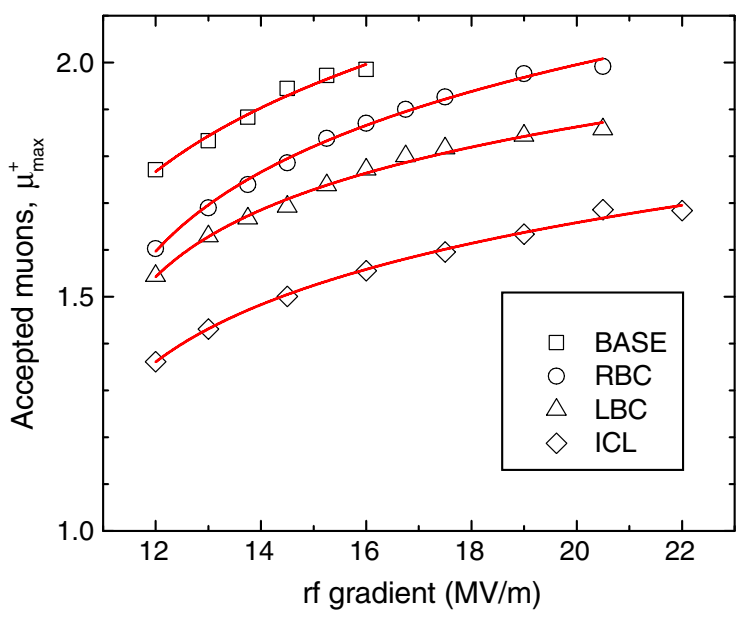

FIG. 11. The degradation of cooling performance with decreased rf voltage. The vertical axis shows the maximum accepted muon yield within a transverse acceptance of $\mathrm{A}_{\mathrm{T}} \leq$ $30 \mathrm{~mm}$ and longitudinal acceptance of $A_{L} \leq 150 \mathrm{~mm}$. The nominal $\mathrm{rf}$ gradient for all cooling scenarios is $16 \mathrm{MV} / \mathrm{m}$.

the loss of performance will not exceed 5\%. On the other hand, if $16 \mathrm{MV} / \mathrm{m}$ can be achieved only in subtesla magnetic fields ( $\mathrm{B} \sim 0.5 \mathrm{~T}$ or less) then the ICL lattice becomes the most viable choice. Accordingly, this will result in a 15\%-20\% particle loss compared to the initially anticipated performance.

\section{SUMMARY}

Beams of accelerated muons are potentially of great interest for fundamental high-energy physics research as well as for medical science, material science, and industrial applications. Since most of the created muons have diffuse energies and are spread in all directions from the target, there is a significant need to cool the beam. In this study we designed, simulated, and compared four different cooling schemes based on ionization cooling. We numerically examined the impact of rf gradient limitations on the overall performance and reviewed important lattice parameters such as the coil configurations, the required magnetic fields, the number of rf cavities, and the absorber lengths for each cooling scheme.

In this study we discussed in more detail novel lattice designs that allow the operation of a rf cavity within subtesla magnetic fields. In order to cross-check our results we employed two independent codes that fully included all basic particle-matter-interaction physical processes such as energy loss, scattering, straggling and muon decay. The two codes showed a very good agreement for all cooling scenarios examined. We showed that by using an ionization cooling channel with radial bucked coils not only is the magnetic field on the cavity iris reduced by a factor of 3 but at the same time the achieved muon yield is only 5\% lower than that of the baseline cooling channel. 


\section{ACKNOWLEDGMENTS}

The authors are grateful to J. S. Berg, X. Ding, H. Kirk, R. B. Palmer, R. Ryne, and H. Witte for many useful discussions. This work is supported by the U.S. Department of Energy, Contract No. DE-AC0298CH10886.

[1] A. Hassenein, S. A. Kahn, B. J. King et al., Proceedings of the 2001 Particle Accelerator Conference, Chicago (IEEE, Piscataway, NJ, 2001), p. 1583.

[2] L. J. Schultz, K. N. Borozdin, J. J. Gomez, G. E. Hogan, J. A. McGill, C. L. Morris, W. C. Priedhorsky, A. Saunders, and M. E. Teasdale, Nucl. Instrum. Methods Phys. Res., Sect. A 519, 687 (2004).

[3] K. Nagamine, K. Shimomura, K. Imai, and J. S. Schultz, Physica (Amsterdam) 374B-375B, 444 (2006).

[4] S. S. Kurennoy, A. J. Jason, and H. Miyadera, in Proceedings of the International Particle Accelerator Conference, Kyoto, Japan (ICR, Kyoto, 2010), p. 3518.

[5] S. Geer, Phys. Rev. D 576989 (1998).

[6] C. M. Ankenbrandt et al., Phys. Rev. ST Accel. Beams 2, 081001 (1999).

[7] H. G. Kirk, S. A. Kahn, H. Ludewig et al., in Proceedings of the 21 st Particle Accelerator Conference, Knoxville, TN, 2005 (IEEE, Piscataway, NJ, 2005), p. 3745.

[8] D. Neuffer, Part. Accel. 14, 75 (1983).

[9] D. Neuffer, Nucl. Instrum. Methods Phys. Res., Sect. A 532, 26 (2004).

[10] Y. Derbenev and R. P. Johnson, Phys. Rev. ST Accel. Beams 8, 041002 (2005).

[11] K. Yonehara, R. P. Johnson, M. Neubauer, and Y.S. Derbenev, in Proceedings of the International Particle Accelerator Conference, Kyoto, Japan (ICR, Kyoto, 2010), p. 870.

[12] M. Chung, M. G. Collura, G. Flanagan et al., Phys. Rev. Lett. 111, 184802 (2013).

[13] R. C. Fernow, J. C. Gallardo, H. G. Kirk, and R. B. Palmer, AIP Conf. Proc. 472, 233 (1999).

[14] J. Monroe, P. Spentzouris, V. Balbekov, P. Lebrun, G. Penn, C. Kim, E. Kim, and D. Kaplan, Phys. Rev. ST Accel. Beams 4, 041301 (2001).

[15] V. Balbekov, Neutrino Factory/ Muon Collider Document No. 204, 2001.

[16] M. Apollonio, J. S. Berg, A. Blondel et al., JINST 4, P07001 (2009).

[17] A. Moretti, Z. Qian, J. Norem, Y. Torun, D. Li, and M. Zisman, Phys. Rev. ST Accel. Beams 8072001 (2005).

[18] D. Stratakis, J. C. Gallardo, and R. B. Palmer, Nucl. Instrum. Methods Phys. Res., Sect. A 620, 147 (2010).
[19] R. B. Palmer, R. C. Fernow, J. C. Gallardo, D. Stratakis, and D. Li, Phys. Rev. ST Accel. Beams 12031002 (2009).

[20] A. Alekou, Ph.D. Dissertation, Imperial College London, 2012.

[21] A. W. Chao and M. Tigner, Handbook of Accelerator Physics and Engineering (World Scientific, Singapore, 1999), p. 176.

[22] R. C. Fernow and R. B. Palmer, Phys. Rev. ST Accel. Beams 10, 064001 (2007).

[23] R. C. Fernow, J. S. Berg, J. C. Gallardo, H. G. Kirk, R. B. Palmer, D. Neuffer, and K. Paul, Nucl. Phys. B, Proc. Suppl. 149, 274 (2005).

[24] K. Yonehara, J. Phys. Conf. Ser. 408, 012062 (2013).

[25] Y. Torun et al., in Proceedings of the International Particle Accelerator Conference, Kyoto, Japan (ICR, Kyoto, 2010), p. 3795.

[26] A. Moretti (private communication).

[27] M. R. Jana, M. Chung, M. Leonova et al., Proceedings of PAC 2013, Pasadena, CA, 2013, p. 1007, http://accelconf .web.cern.ch/AccelConf/pac2013/papers/wepma12.pdf.

[28] C. T. Rogers, D. Stratakis, G. Prior, S. Gilardoni, D. Neuffer, P. Snopok, A. Alekou, and J. Pasternak, Phys. Rev. ST Accel. Beams 16, 040104 (2013).

[29] A. Alekou and J. Pasternak, JINST 7, P08017 (2012).

[30] http://fs.magnet.fsu.edu/ lee/plot/plot.htm.

[31] Martin N. Wilson, Superconducting Magnets (Oxford University Press, Oxford, 1983), p. 55.

[32] F. Herlach and N. Miura, High Magnetic Fields: Science and Technology (World Scientific, Singapore, 2003), Vol. 3.

[33] C. T. Rogers, in Neutrino Factories, Superbeams and Beta Beams: 11th International Workshop on Neutrino Factories, Superbeams and Beta Beams-NuFact09, edited by M. C. Goodman, D. M. Kaplan, and Z. Sullivan (AIP Press, Melville, NY, 2010), pp. 298-302.

[34] R. C. Fernow, in Proceedings of the 21st Particle Accelerator Conference, Knoxville, TN, 2005 (IEEE, Piscataway, NJ, 2005), p. 2651.

[35] T. J. Roberts et al., in Proceedings of the 11th European Particle Accelerator Conference, Genoa, 2008 (EPS-AG, Genoa, Italy, 2008), p. 2776.

[36] map.fnal.gov.

[37] D. Li et al., J. Phys. G 29, 1683 (2003).

[38] See Eq. (16) in R. C. Fernow, Neutrino Factory/Muon Collider Document No. 280 (2003).

[39] J. C. Lagarias, J. A. Reeds, M. H. Wright, and P. E. Wright, SIAM J. Optim. 9112 (1998)

[40] H. K. Sayed, J. S. Berg, H. G. Kirk et al., Proceedings of PAC 2013, Pasadena, CA, 2013, p. 547, http://accelconf .web.cern.ch/AccelConf/pac2013/papers/tupba11.pdf.

[41] E. Wildner, A. Kurup, C. Densham and F. J. P. Soler, EUROnu Costing Report, EUROnu-WP1-5, 2012 [http:// www.euronu.org]. 